\title{
A NOTE ON A FUNCTIONAL INEQUALITY
}

\author{
HORST ALZER \\ Department of Pure Mathematics \\ Applies Mathematics and Astronomy \\ University of South Africa \\ P.O. Box 392 \\ 0001 Pretoria, South Africa
}

(Received September 25, 1990 and in revised form March 5, 1991) ABSTRACT. We prove: If $r_{1}, \ldots, r_{k}$ are (fixed) positive real numbers with $\prod_{j=1}^{k} r_{j}>1$, then the only
entire solutions $\varphi: C \rightarrow C$ of the functional inequality

$$
\prod_{j=1}^{k}|\varphi(r z)| \geq\left(\prod_{j=1}^{k} r_{j}\right)|\varphi(z)|^{k}
$$

are $\varphi(z)=c z^{n}$, where $c$ is a complex number and $n$ i s a positive integer.

KEY WORDS AND PHRASES. Functional inequality, entire functions.

1991 AMS SUBJECT CLASSIFICATION CODE. 39C05.

1. INTRODUCTION.

Inspired by a problem of $\mathrm{H}$. Haruki, who asked for all entire solutions of

$$
|\varphi(z+w)|^{2}+|\varphi(z-w)|^{2}+2|\varphi(0)|^{2} \geq 2|\varphi(z)|^{2}+2|\varphi(w)|^{2},
$$

J. Walorski [1] proved in 1987 the following interesting proposition:

Let $r>1$ be a (fixed) real number. Then the only entire solutions $\varphi: C \rightarrow C$ of the functional inequality

$$
|\varphi(r z)| \geq r|\varphi(z)|
$$

are

$$
\varphi(z)=c z^{n}
$$

where $c \in \mathbf{C}$ and $n \in \mathbb{N}$.

As an application of this theorem, Walorski showed that the only entire functions $\varphi: C \rightarrow C$ satisfying (1.1) and $\varphi(0)=0$ are the monomials (1.2). The aim of this note is to prove an extension of Walorski's result by using a method which is (slightly) different from the two approaches presented in [1].

2. MAIN RESULTS.

Theorem. Let $r_{1}, \ldots, r_{k}$ be (fixed) positive real numbers with $\prod_{j=1}^{k} r_{j}>1$. Then the only entire 
solutions $\varphi: C \rightarrow C$ of

$$
\prod_{j=1}^{k}\left|\varphi\left(r_{j}^{z}\right)\right| \geq\left(\prod_{j=1}^{k} r_{j}\right)|\varphi(z)|^{k}
$$

are the functions $\varphi(z)+c z^{n}$, where $c$ is a complex number and $n$ is a positive integer.

PROOF. Simple calculations reveal that the functions $\varphi(z)=c z^{n}(c \in \mathbf{C}, n \in \mathbb{N})$ satisfy (2.1). Next we assume that $\varphi$ is an entire solution of inequality (2.1).

Because of $\prod_{j=1}^{k} r_{j}>1$ we conclude from (2.1) with $z=0$ that $\varphi$ has at 0 a zero. Let $n$ be the order of this zero; we define

$$
f(z)=\varphi(z) / z^{n}
$$

then $f$ is an entire function with $f(0) \neq 0$. From (2.1) we obtain

$$
\prod_{j=1}^{k}\left|f\left(r_{j}^{z}\right)\right| \geq\left(\prod_{j=1}^{k} r_{j}^{1-n}\right)|f(z)|^{k} .
$$

We suppose that $f$ has a zero $z_{0}$. By induction it follows from (2.3) that $z_{0} / r_{1}^{m}$ is a root of $f$ for all non-negative integers $m$. From the identity theorem we conclude $f(z) \equiv 0$ which contradicts the condition $f(0) \neq 0$. Hence $f$ has no zero which implies that the function

is entire. From (2.3) we conclude

$$
g(z)=\frac{f(z)^{k}}{\prod_{j=1}^{k} f(r z)}
$$

$$
|g(z)| \leq \prod_{j=1}^{k} r_{j}^{n-1} \quad \text { for all } z \in C,
$$

and Liouville's theorem implies that $g$ is a constant. Therefore we have

$$
f(z)^{k}=K \prod_{j=1}^{k} f(r z), \quad K \in \mathbf{C} .
$$

Since $f(0) \neq 0$ we get from $(2.5): K=1$;

hence

$$
f(z)^{k}=\prod_{j=1}^{k} f(r z)
$$

Differentiation leads to

$$
k \frac{f^{\prime}(z)}{f(z)}=\sum_{j=1}^{k} r_{j} \frac{f^{\prime}\left(r_{j}^{z}\right)}{f\left(r_{j} z\right)}
$$

Setting

$$
\frac{f^{\prime}(z)}{f(z)}=\sum_{m=0}^{\infty} a_{m} z^{m}
$$

we obtain from (2.7) and (2.8):

$$
\sum_{m=0}^{\infty} k a_{m} z^{m}=\sum_{m=0}^{\infty}\left(a_{m} \sum_{j=1}^{k} r_{j}^{m+1}\right) z^{m}
$$


and comparing the coefficients of $z^{m}$ yields for all $m \geq 0$ :

$$
k a_{m}=a_{m} \sum_{j=1}^{k} r_{j}^{m+1} .
$$

We assume that there exists an integer $m_{0} \geq 0$ such that $a_{m_{0}} \neq 0$, then we get from the arithmetic mean-geometric mean inequality and from (2.10):

$$
\left[\sum_{j=1}^{k} r_{j}^{m_{0}+1}\right]^{1 / k} \leq \frac{1}{k} \sum_{j=1}^{k} r_{j}^{m_{0}+1}=1
$$

which contradicts the assumption $\sum_{j=1}^{k} r_{j}>1$. Hence, $a_{m}=0$ for all $m>0$. This implies that $f$ is a constant, say $c \in \mathbf{C}$, and therefore we obtain $\varphi(z)=c z^{n}$.

It is natural to look for all entire functions $\varphi: C \rightarrow C$ which satisfy the following additive counterpart of inequality (2.1):

$$
\left(\sum_{j=1}^{k} \varphi(r z)\right) \geq \sum_{j=1}^{k} r_{j}|\varphi(z)|
$$

where $r_{1}, \ldots r_{k}$ are (fixed) positive real numbers with $\sum_{j=1}^{k} r_{j}>k$. The monomials $\varphi(z)=c z^{n}(c \in \mathbb{C}, n \in \mathbb{N})$ are solutions of (2.11). Indeed, inequality (2.11) with $\varphi(z)=c z^{n}$ reduces to

$$
\sum_{j=1}^{k} r_{j}^{n} \geq \sum_{j=1}^{k} r_{j}
$$

which follows immediately from Jensen's inequality and the assumption $\sum_{j=1}^{k} r_{j}>k$. By an argumentation similar to the one we have used to establish the theorem it can be shown that the functions $\varphi(z) c z^{n}(c \in \mathbb{C}, n \in \mathbb{N})$ are the only entire solutions of (2.11). This provides another extension of Walorski's result.

If the expression on the left-hand side of $(2.11)$ will be replaced by $\sum_{j=1}^{k}\left|\varphi\left(r_{j}\right)\right|$, then we
clude from the triangle inequality that $\varphi(z)=c z^{n}(c \in \mathbb{C}, n \in \mathbb{N})$ also solve

$$
\sum_{j=1}^{k}\left|\varphi(r z) \geq \sum_{j=1}^{k} r_{j}\right| \varphi(z) \mid
$$
where $r_{1}, \ldots, r_{k}$ are (fixed) positive real numbers with $\sum_{j=1}^{k} r_{j}>k$. We finish by asking: Are there
more solutions of $(2.13)($ if $k>1)$ ?

\section{REFERENCE}

1. WALORSKI, J., On a functional inequality, Aequationes Math. $\underline{32}$ (1987), 213-215. 


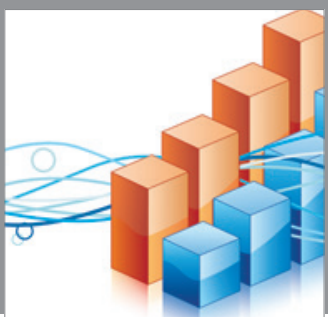

Advances in

Operations Research

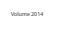

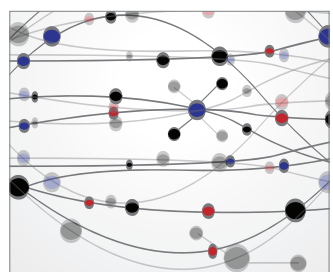

\section{The Scientific} World Journal
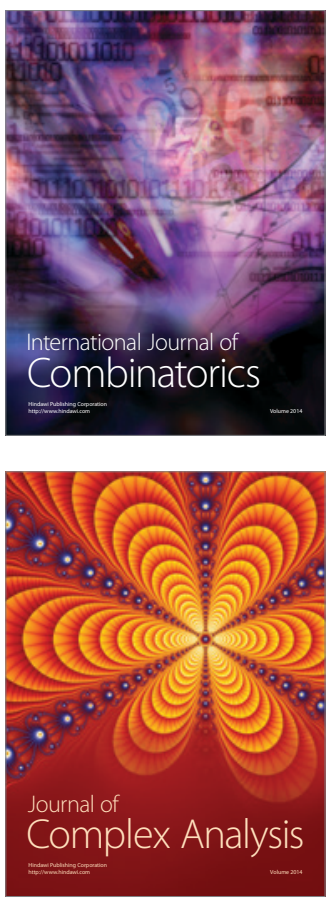

International Journal of

Mathematics and

Mathematical

Sciences
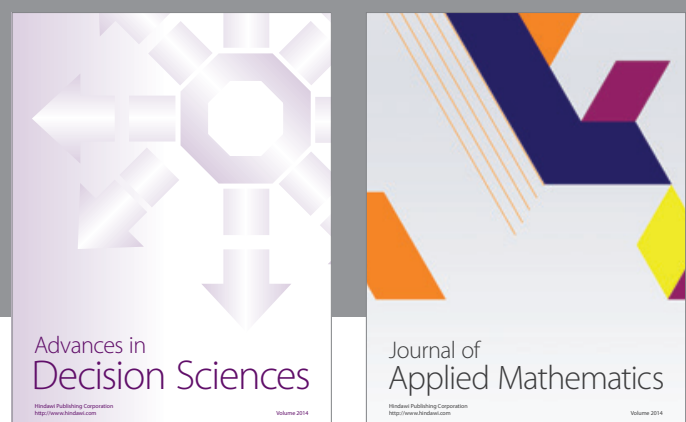

Journal of

Applied Mathematics
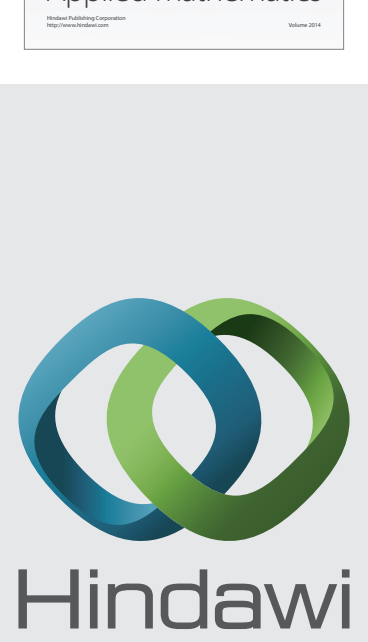

Submit your manuscripts at http://www.hindawi.com
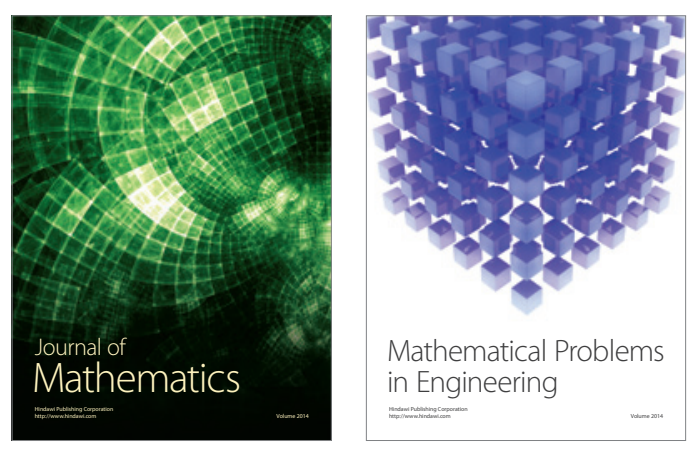

Mathematical Problems in Engineering
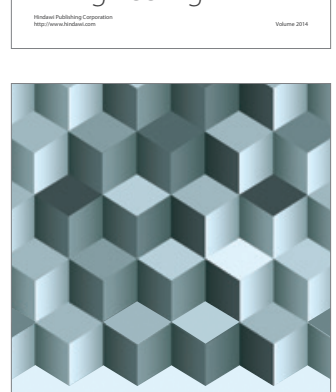

Journal of

Function Spaces
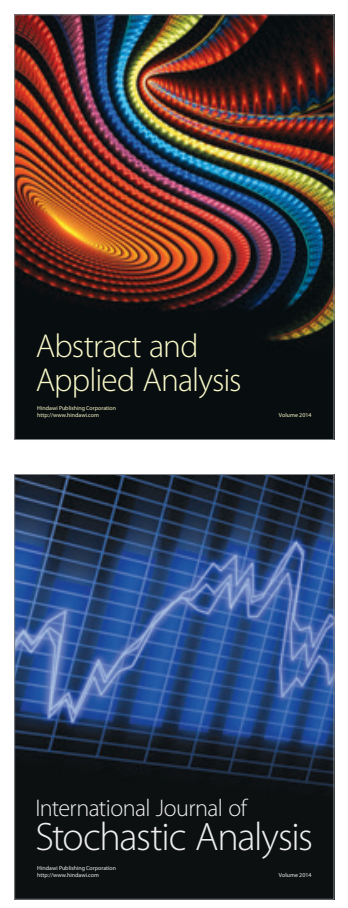

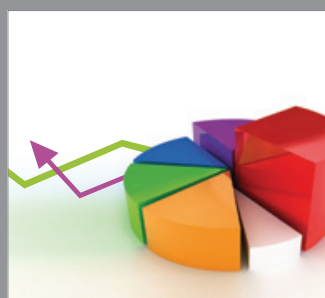

ournal of

Probability and Statistics

Promensencen
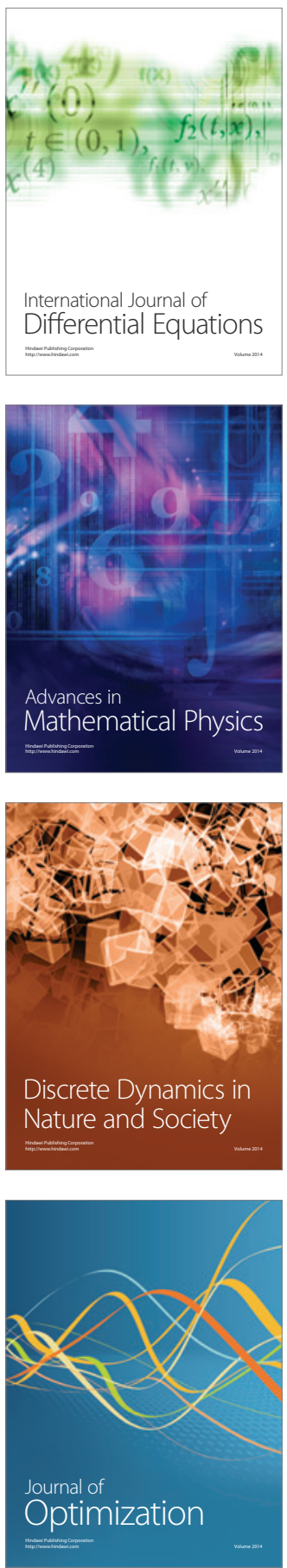